\title{
Ce que révèlent les discours des acteurs institutionnels sur un « au-delà du PIB »
}

\author{
Géraldine Thiry ${ }^{1}$, Léa Sébastien ${ }^{2}$, Tom Bauler ${ }^{3}$ \\ 1 Économie, Université catholique de Louvain, IRES, CIRTES, 1348 Louvain-la-Neuve, Belgique \\ 2 Géographie humaine, Université Toulouse II, CNRS, Laboratoire GEODE, 31100 Toulouse cedex 9, France \\ 3 Économie écologique, Université libre de Bruxelles, Chaire Environnement et économie, IGEAT, 1050 Bruxelles, Belgique
}

Le produit intérieur brut (PIB), qui est l'un des indicateurs phares de la comptabilité nationale, fait régulièrement l'objet de débats quant à la relation existant entre la croissance économique et le progrès social. Par construction, en effet, le PIB ne reflète pas nombre de transformations du monde, qu'il s'agisse d'environnement, de santé des populations ou de l'état des savoirs. Les réflexions sur son éventuelle amélioration ou la nécessité de se référer à d'autres indicateurs sociaux ou environnementaux se sont multipliées depuis une quinzaine d'années. Dans le cadre d'un projet européen, les auteurs ont interrogé des acteurs institutionnels, des politiques, des administratifs, des économistes, des statisticiens de différents pays de l'OCDE qui sont, de près ou de loin, concernés par cette réflexion. Leur but est de comprendre si, oui ou non, l'idée d'un « audelà du PIB » constitue pour ces acteurs un levier effectif de transformation vers une société post-croissance.

La Rédaction

\section{Mots-clés :} environnement ; gouvernance ; au-delà du PIB ; indicateurs de développement durable ; analyse de discours

\section{Keywords:}

environment; governance; beyond GDP; sustainability indicators; discourse analysis
Résumé - L'objectif d'un « au-delà du PIB » mobilise aujourd'hui de nombreux acteurs. Si les réflexions pionnières se sont d'abord cantonnées aux milieux écologistes, à la société civile organisée et aux politiques locales, elles ont récemment intégré les sphères institutionnelles nationales et internationales. Mais de quoi l'intérêt des acteurs institutionnels pour cette question est-il le signe ? Un " au-delà du PIB » serait-il un objectif rhétorique par défaut, en l'absence de stratégie crédible de sortie de crise, ou le signe d'un véritable changement paradigmatique? L'article entend élucider ces questions par l'analyse des discours d'acteurs institutionnels sur un « au-delà du PIB ». Les débats institutionnels, bien qu'intégrant de nouveaux enjeux comme la soutenabilité et le bien-être, restent dominés par des intérêts et contraintes de court terme, dont la croissance du PIB demeure un élément central. Derrière l'illusion démocratique, les ambiguïtés conceptuelles liées à un « au-delà du PIB » tendent à asseoir un rapport de force existant.

Auteur correspondant : G. Thiry, geraldine.thiry@uclouvain.be 
De nombreux travaux scientifiques contribuent, depuis plus de quarante ans, à montrer les limites écologiques et sociales d'un modèle de société fondé sur la poursuite infinie de la croissance du Produit intérieur brut (PIB) (Meadows et al., 1972 ; Jackson, 2010 ; Caminel et al., 2014 ; Steffen et al., 2015). Si les réflexions pionnières sur la croissance et son indicateur phare se sont d'abord cantonnées aux milieux écologistes et militants, aux sphères de la société civile organisée et aux politiques locales (Méda, 2000 ; Gadrey et Jany-Catrice, 2012), elles ont récemment intégré les sphères institutionnelles nationales et internationales (Stiglitz et al., 2009; Hák et al., 2012 ; Chancel et al., 2014) ${ }^{1}$. Aujourd'hui, sous l'apparente homogénéité d'un « au-delà du PIB », coexistent de nombreuses initiatives dont les motifs, les procédures et les finalités divergent et sont parfois antagonistes. Comme le montre Pestre (2011, p. 31) à propos du développement durable, l'idée d'un "au-delà du PIB » relève " $d$ 'un syntagme aux usages très divers, $d$ 'un ensemble de convictions, de pratiques et $\mathrm{d}^{\prime}$ institutions chargées de sens variables, [...] d'un enjeu autour duquel se déroule un débat, une bataille politique entre projets de société ».

Dans cette nébuleuse, les sphères institutionnelles, dotées d'importants leviers opérationnels et politiques, sont amenées à jouer un rôle important dans la diffusion de nouvelles conventions de richesse et dans l'orientation que pourrait prendre un "au-delà du PIB ». Il nous semble dès lors essentiel de comprendre ce que révèle l'intérêt croissant de nombreux acteurs institutionnels pour cette question. Nous entendons évaluer la mesure dans laquelle les tentatives actuelles d'un dépassement du PIB au sein des sphères institutionnelles sont de nature à catalyser un changement de " référentiel ».

Le référentiel est entendu ici au sens que lui donne Pierre Muller, comme une représentation générale, constituée d'un ensemble de valeurs fondamentales qui renvoient aux croyances de base d'une société, ainsi que des normes qui permettent de choisir entre des conduites (Muller, 2010). Au cours du XX ${ }^{\mathrm{e}}$ siècle, Muller (1992, 2010) identifie trois référentiels globaux successifs : le référentiel « libéral » dominant jusqu'à la crise de 1929, le référentiel de «l'État Providence » jusqu'à la fin des années 1970, puis le référentiel « de marché », caractérisé par une redéfinition de la frontière public-privé, où l'action de l'État, recentrée sur des fonctions de régulation économique, est évaluée selon des normes d'efficience calquées sur des modes de gestion privée. Comme le

\footnotetext{
1 Un nombre important d'indicateurs a ainsi vu le jour depuis trente ans, parmi lesquels l'indice de santé sociale (1987), l'indice de développement humain (1990), l'empreinte écologique (1994), l'indice de progrès véritable (1994), l'indicateur de bien-être économique (1998), l'épargne nette ajustée, le Happy Planet Index (2006), l'indice de richesse inclusive (2012) ou, plus récemment, l'indice de progrès social (2013).
}

montre Méda (2013), il semble que l'accroissement de la valeur produite (ajoutée) par l'économie transcende ces trois configurations sociohistoriques et constitue dans chacune d'elles un principe central du fonctionnement de l'économie. La mise en question du PIB marquerait-elle alors l'esquisse d'une rupture inédite vis-à-vis de cet « invariant » - la croissance - qui relie chacun des trois référentiels du $X X^{\mathrm{e}}$ siècle, et donc vis-à-vis du référentiel actuel ?

Pour y répondre, nous analysons les discours de plusieurs acteurs institutionnels. Lors de leur collecte, ces discours ont été organisés suivant trois dimensions : les savoirs, les représentations et les pratiques, dans la lignée de ce que proposent Donnadieu et Karsky (2002). La combinaison de ces éléments de discours constitue le matériau à partir duquel nous formulons par la suite une analyse mobilisant le concept de « référentiel ».

L'article est structuré comme suit. La deuxième section présente les acteurs rencontrés et la méthodologie utilisée pour collecter les discours. La troisième section présente les principaux éléments de discours (savoirs, représentations et pratiques) récoltés. La quatrième section analyse les positionnements des acteurs institutionnels pour comprendre si un changement de référentiel est à l'œuvre et évalue la mesure dans laquelle la croissance économique est questionnée. La cinquième et dernière partie conclut.

\section{Acteurs rencontrés et méthodologie}

Le projet européen BRAINPOoL ${ }^{2}$, mené entre octobre 2011 et mars 2014, nous a permis de récolter un large matériau empirique ${ }^{3}$. L'objectif de ce projet était de renforcer et d'accélérer le développement et l'usage effectif de nouveaux indicateurs susceptibles de contrebalancer le PIB, afin de soutenir les politiques de développement durable de l'Union européenne. C'est pourquoi, dans le cadre de ce projet, nous avons été amenés à étudier les facteurs d'usage et de non-usage de nouveaux indicateurs au sein des sphères institutionnelles: au niveau de l'Union européenne, de l'Organisation de coopération et de développement économique (OCDE) et dans cinq pays européens.

Nous avons approché à la fois différents acteurs impliqués et non impliqués dans la recherche ou l'élaboration de nouveaux indicateurs (voir Encadré). Les acteurs

\footnotetext{
2 Voir www.brainpoolproject.eu pour le détail de la méthodologie et les résultats complets de l'étude.

$3 \mathrm{Qu}$ 'ils soient statisticiens, politiques ou administratifs, nous qualifions les acteurs rencontrés « d'institutionnels » pour les distinguer clairement des sphères de la société civile organisée, dont les représentants rencontrés dans le cadre de notre étude ne constituent qu'une très faible part.
} 


\begin{abstract}
Encadré. Choix des acteurs rencontrés et modalités de rencontres.
Dans le choix des acteurs rencontrés, nous n'avons pas visé la représentativité sociologique. Nous avons en revanche été attentifs à l'incidence potentielle des positionnements des acteurs rencontrés qui, par leur fonction ou leur implication, sont susceptibles d'avoir un poids décisionnel non négligeable dans l'adoption (ou la non-adoption) de nouveaux indicateurs.

Empiriquement, les acteurs « impliqués » ont été interrogés dans le cadre d'entretiens individuels. Au total, 36 entretiens ont été menés dans deux contextes particuliers : 21 entretiens en France et 15 durant le 4 e forum mondial de l'OCDE, « Mesurer le bien-être pour les politiques de développement » (New Delhi, 16-19 octobre 2013). La France fait l'objet d'une attention particulière : tant nos analyses institutionnelles que les rencontres d'acteurs officiels témoignent de l'influence considérable du Rapport Stiglitz (Stiglitz et al., 2009) dans les représentations des acteurs. Quant au forum mondial de l'OCDE, il représentait l'opportunité de voir rassemblés en un même lieu un grand nombre d'acteurs institutionnels impliqués dans l'élaboration, la diffusion ou l'utilisation de nouveaux indicateurs. Les entretiens ont été conduits selon un guide semi-directif structuré en trois grands thèmes :1) les facteurs stimulant l'utilisation des nouveaux indicateurs " au-delà du PIB »; 2) la (non-) demande politique pour de nouveaux indicateurs ; 3 ) le rôle (conceptuel, instrumental, politique) des indicateurs dans la société. Les tableaux 1 et 2 présentent les institutions et les fonctions des acteurs rencontrés au cours des entretiens individuels ${ }^{1}$.

Parallèlement, quatre ateliers ont été organisés dans différents pays européens, principalement à destination des acteurs « non impliqués ». Ils ont eu lieu en République tchèque, en France, au Royaume-Uni et en Allemagne. Ces quatre pays ont été choisis car ils constituaient les cas d'étude nationaux au sein de BRAINPOoL. Chaque atelier a été divisé en trois moments distincts : 1) l'agenda « au-delà du PIB »;2) les indicateurs « au-delà du PIB » ; 3) le modèle « au-delà du PIB ». Chaque moment comprenait un exposé instructif sur l'état des débats et un temps de discussion avec les participants. Les tableaux 3 à 6 présentent les institutions et les fonctions des acteurs rencontrés lors des ateliers.
\end{abstract}

Le détail du guide d'entretien peut être obtenu sur demande aux auteurs.

dits « impliqués » ont été identifiés par leurs travaux, initiatives ou décisions, menés dans le sens d'un élargissement des cadres réflexifs et comptables, avec l'objectif d'améliorer la prise en compte d'enjeux globaux de bienêtre et/ou de soutenabilité. Les acteurs «non impliqués » évoluent dans les mêmes sphères que les acteurs impliqués, sont également potentiellement confrontés aux limites du PIB, mais aucun de leurs travaux, initiatives ou décisions n'a fait l'objet d'une réflexion sur la nécessité $\mathrm{d}$ 'aller au-delà du PIB. La distinction que nous avons établie a priori entre ces deux communautés de pratiques sera discutée, au regard de nos résultats, en fin d'article.

\section{Éléments de discours : savoirs, représentations, pratiques}

Les éléments de discours récoltés lors des entretiens et ateliers ont été organisés, sur la base de la grille que proposent Donnadieu et Karsky (2002), en «savoirs " (connaissances de type scientifique, institutionnel ou vernaculaire que mobilise l'acteur lors de l'entretien), « représentations" (perceptions, valeurs morales, opinions exprimées par l'acteur) et «pratiques» (actions, réalisations ou projets dans lesquels s'inscrit l'acteur). Nous présentons ci-dessous notre appréciation de l'état des savoirs, des représentations et des pratiques reliés à l'agenda «au-delà du PIB » ${ }^{4}$. Ces éléments de discours constituent la base de l'analyse que nous effectuerons dans la section suivante, afin de comprendre dans quelle mesure un changement de « référentiel » est (ou non) à l'œuvre auprès des acteurs institutionnels.

\section{Des savoirs faibles}

Les entretiens et ateliers suggèrent un état des savoirs relativement faible quant aux lacunes du PIB et aux alternatives mises en débat.

\section{Critiques du PIB peu connues}

Dans la plupart des ateliers menés, les acteurs non impliqués dans un " au-delà du PIB », bien qu'ils s'accordent sur le caractère imparfait du PIB, ont des connaissances superficielles des critiques formulées à son égard ${ }^{5}$.

\footnotetext{
4 Notons que la section dédiée aux représentations est sensiblement plus longue que les deux autres, du fait de notre méthodologie : les entretiens et ateliers ayant pour objet initial de faire s'exprimer les acteurs sur leurs visions d'un « au-delà $\mathrm{du}$ PIB », ils comportent un biais en faveur des représentations.

5 Nous avons interrogé tous les acteurs sur ce qu'ils savaient de certains éléments de débat à l'égard du PIB (entre autres : les enjeux de la monétisation ; les implications des indicateurs composites $v s$ les tableaux de bord ; les indicateurs objectifs vs subjectifs ; le débat croissance $v s$ décroissance).
} 
Tableau 1. Acteurs rencontrés en entretiens individuels en France.

\begin{tabular}{|c|c|}
\hline Ministère de l'Économie, des Finances et de l'Industrie & Responsable de la mission stratégie \\
\hline Ministère des Affaires étrangères & $\begin{array}{l}\text { Directeur adjoint de l'économie globale et des stratégies du } \\
\text { développement }\end{array}$ \\
\hline \multirow[t]{5}{*}{ Ministère du Développement durable } & $\begin{array}{l}\text { Chef de la délégation au développement durable, Commissa- } \\
\text { riat général au développement durable (CGDD) }\end{array}$ \\
\hline & $\begin{array}{l}\text { Service statistique, Conseil national de l'information statistique } \\
\text { (CNIS, ancien Insee) }\end{array}$ \\
\hline & $\begin{array}{l}\text { Inspecteur du Conseil général de l'environnement et du déve- } \\
\text { loppement durable }\end{array}$ \\
\hline & $\begin{array}{l}\text { Ancien responsable mission prospective + Institut français de } \\
\text { l'environnement (IFEN) }\end{array}$ \\
\hline & $\begin{array}{l}\text { Commission spéciale Développement durable, Conseil général } \\
\text { de l'environnement et du développement durable (CGEDD) }\end{array}$ \\
\hline \multirow[t]{3}{*}{ Insee } & $\begin{array}{l}\text { Responsable du département des études économiques } \\
\text { d'ensemble }\end{array}$ \\
\hline & Chef de la division conditions de vie des ménages \\
\hline & Statisticien au département de la coordination statistique \\
\hline $\begin{array}{l}\text { Insee / Commission sur la mesure des performances } \\
\text { économiques et du progrès social }\end{array}$ & $\begin{array}{l}\text { Ancien responsable du département des études économiques } \\
\text { d'ensemble - Actuel rédacteur en chef économie et statistique }\end{array}$ \\
\hline Conseil économique, social et environnemental (CESE) & $\begin{array}{l}\text { Secrétaire confédéral, Confédération française démocratique } \\
\text { du travail (CFDT) }\end{array}$ \\
\hline Conseil d'analyse économique & Secrétaire général \\
\hline Bureau d'information et de prévisions économiques (BIPE) & Associé et vice-président du BIPE - coordinateur du rapport \\
\hline $\begin{array}{l}\text { Forum pour d'autres indicateurs de richesse (FAIR) / Commis- } \\
\text { sion Stiglitz }\end{array}$ & Professeur d'économie \\
\hline Parlement européen & $\begin{array}{l}\text { Député vert du Parlement européen, ancien journaliste du } \\
\text { Monde }\end{array}$ \\
\hline $\begin{array}{l}\text { Organisation de coopération et de développement économique } \\
(\mathrm{OCDE})\end{array}$ & Économiste \\
\hline Alternatives économiques & Rédacteur en chef adjoint \\
\hline Association de comptabilité nationale & Président \\
\hline Région Nord-Pas-de-Calais & $\begin{array}{l}\text { Responsable du rapport de l'Association des régions de France } \\
\text { sur les nouveaux indicateurs }\end{array}$ \\
\hline ATD Quart Monde & Coprésident \\
\hline Conseil d'analyse stratégique & Chef du département économie \\
\hline
\end{tabular}

Deux observations sont récurrentes : les acteurs pointent le décalage entre les évolutions respectives du bien-être subjectif et du PIB d'une part, et mentionnent les dégâts environnementaux de la croissance d'autre part. Les critiques, plus précises, généralement formulées sur l'information que véhicule le PIB quand il est interprété comme indicateur de bien-être (Gadrey et Jany-Catrice, 2012 ; Stiglitz et al., 2009) ne sont pas évoquées.

\section{Initiatives alternatives et complémentaires méconnues}

On observe, parmi les deux familles d'acteurs ( «impliqués »et « non impliqués »), une méconnaissance des alternatives et compléments au PIB. Chez les acteurs extérieurs, cette méconnaissance n'est pas surprenante. Auprès des acteurs impliqués, la multiplicité des initiatives et, pour certaines d'entre elles, le manque de médiatisation à large échelle entraînent l'absence d'une vision 
Tableau 2. Acteurs rencontrés en entretiens individuels lors du forum mondial de l'OCDE.

\begin{tabular}{|l|l|}
\hline Université de Delhi (Inde) & Professeur \\
\hline $\begin{array}{l}\text { Oxford Poverty and Human Development Initiative } \\
\text { (Royaume-Uni) }\end{array}$ & Professeur \\
\hline Conseil économique et social, Luxembourg & Vice-président \\
\hline Direction de la statistique, OCDE & Directeur du programme « Better Life Index » \\
\hline Applied Survey Research (États-Unis) & Président \\
\hline Agence des droits fondamentaux de l'Union européenne & Chef du département « Égalité et droits des citoyens » \\
\hline Trade Union Advisory Committee to the OECD & Secrétaire général \\
\hline Bureau national de statistique (Royaume-Uni) & Statisticien, directeur du programme « National Well-Being » \\
\hline DG Environnement, Commission européenne & Directeur général \\
\hline Commission Stiglitz & Coprésident \\
\hline Institut national de statistique italien & Statisticien, président de l'institut \\
\hline Human Development Report Office, UNDP & $\begin{array}{l}\text { Responsable de l'équipe en charge du rapport sur le dévelop- } \\
\text { pement humain }\end{array}$ \\
\hline Direction de l'emploi, du travail et des affaires sociales, OCDE & Économiste \\
\hline Institut de recherche sur le développement & Directeur de recherche \\
\hline Institut national de statistique, Israël & Statisticien, en charge du programme soutenabilité \\
\hline
\end{tabular}

Tableau 3. Acteurs présents en ateliers en Allemagne.

\begin{tabular}{|l|l|}
\hline BMWI (ministère de l'Économie) & Membre du cabinet \\
\hline Die Zeit & Journaliste \\
\hline Die Tagezeitung & Journaliste \\
\hline Ecologic Institute (NB : institution d'accueil de l'atelier) & Chercheur \\
\hline Destatis (Institut allemand de statistiques) & Statisticien \\
\hline
\end{tabular}

Tableau 4. Acteurs présents en ateliers en France.

\begin{tabular}{|l|l|}
\hline Insee & $\begin{array}{l}\text { Administrateur de l'Insee, chef de la division profilage } \\
\text { et traitement des grandes unités }\end{array}$ \\
\hline Deloitte & Associé en charge du secteur public \\
\hline Le Monde & Journaliste, responsable du supplément Monde Économie \\
\hline Fondation Banque de France pour la recherche & Administrateur trésorier \\
\hline CEPII (Centre de recherche en économie internationale) & Économiste (Trade and Globalization) \\
\cline { 2 - 2 } & Économiste (Competitiveness E Growth) \\
\hline $\begin{array}{l}\text { DGCIS (DG compétitivité, industrie et services), ministère } \\
\text { du Redressement productif }\end{array}$ & Responsable de la mission stratégie \\
\hline $\begin{array}{l}\text { Direction générale de la mondialisation, du développe- } \\
\text { ment et des partenariats, ministère des Affaires étrangères } \\
\text { et européennes }\end{array}$ & $\begin{array}{l}\text { Directeur adjoint de l'économie globale et des stratégies } \\
\text { du développement }\end{array}$ \\
\hline $\begin{array}{l}\text { OFCE (Observatoire français des conjonctures écono- } \\
\text { miques) }\end{array}$ & Économiste senior au département des études \\
\hline
\end{tabular}

globale de ce qui existe aujourd'hui en matière d'indicateurs. La seule initiative connue de tous est le rapport Stiglitz. En revanche, les initiatives portées par l'OCDE (par exemple, le « Global Project » ou l'« Indice de mieux vivre ») ou l'Union européenne (par exemple, la conférence « Au-delà du PIB » ou le rapport de la Commission européenne «Le PIB et au-delà ») se sont révélées majoritairement inconnues des participants. Les connaissances 
Tableau 5. Acteurs présents en ateliers en République tchèque.

\begin{tabular}{|l|l|}
\hline $\begin{array}{l}\text { Society for the Sustainable Living } \\
\text { (organisation non gouvernementale) }\end{array}$ & Président de l'organisation \\
\hline Ville de Cheb & Chef du département pour le développement urbain \\
\hline $\begin{array}{l}\text { Agence tchèque de l'information environnementale } \\
\text { (CENIA) }\end{array}$ & $\begin{array}{l}\text { Fonctionnaire - département des systèmes d'information, } \\
\text { des statistiques environnementales }\end{array}$ \\
\hline Ministère de l'Environnement & Fonctionnaire - département des instruments économiques \\
\hline State Environmental Fund (SFZP) & Project manager \\
\hline Ville de Cheb & Maire \\
\hline Institut statistique tchèque (CSO) & $\begin{array}{l}\text { Fonctionnaire - département des statistiques sur l'agricul- } \\
\text { ture, les forêts et l'environnement }\end{array}$ \\
\hline
\end{tabular}

Tableau 6. Acteurs présents en ateliers en Angleterre.

\begin{tabular}{|l|l|}
\hline $\begin{array}{l}\text { UK Department for Business Innovation and Skills (BIS) } \\
\text { (ministère des Affaires et de l'Innovation) }\end{array}$ & $\begin{array}{l}\text { Deux représentants officiels du département } \\
\text { Trois conseillers politiques }\end{array}$ \\
\hline Agence de consultance & Quatre consultants d'affaires \\
\hline Fédération du secteur des affaires (business organisations) & Quatre représentants du secteur des affaires \\
\hline Syndicats & Deux représentants syndicaux \\
\hline Université & Un chercheur dans le secteur des affaires \\
\hline
\end{tabular}

liées à des indicateurs spécifiques sont tout aussi faibles. D'une liste de douze indicateurs composites associés au bien-être ou à la soutenabilité, seuls l'empreinte écologique et l'indice de développement humain étaient connus par tous ${ }^{6}$.

\section{Diversité des représentations}

Deux familles de représentations dominent les discours étudiés. L'une porte sur la place du PIB face aux critiques et aux alternatives ; l'autre sur l'articulation entre bien-être et soutenabilité.

\section{Prépondérance du PIB}

Pour denombreuxacteurs, principalement « non impliqués », le PIB reste un indicateur incontournable. Les raisons invoquées peuvent être présentées comme suit.

\section{Rôle prépondérant du PIB et absence de modèle alternatif}

L'argument récurrent est que le PIB n'a pas été initialement conçu pour englober les enjeux de natures sociale

\footnotetext{
6 Notons que la liste des douze indicateurs n'a été présentée que lors de 18 entretiens sur 36 (dans les entretiens « longs »). En atelier, en revanche, tous les participants ont été invités à se prononcer sur ces douze indicateurs.
}

et écologique ; c'est son usage politique et médiatique qui a généré l'assimilation de sa croissance au progrès des sociétés. La critique qui consiste à dénoncer la non-prise en compte des enjeux sociaux et écologiques dans le PIB est donc considérée comme non fondée. Par ailleurs, au sein et en dehors de la sphère « au-delà du PIB », il n'est pas jugé envisageable d'organiser la vie économique sans le PIB. L'une des principales raisons invoquées est l'absence d'une proposition socialement acceptable, écologiquement désirable, politiquement légitime, théoriquement cohérente et pratiquement réalisable d'un modèle économique alternatif. Beaucoup d'acteurs ont évoqué, à cet égard, le manque de lien entre certains indicateurs mis en débat aujourd'hui et le modèle économique actuel.

\section{Influence ambiguë de la crise dans les représentations}

L'une des positions observées consiste à soutenir que la crise, en mettant en exergue les limites du système, renforcerait le mouvement d'un « au-delà du PIB ». Parallèlement, la plupart des acteurs ont conscience que la crise complique de facto l'émergence d'alternatives concrètes, $\mathrm{du}$ fait des manques de moyens politiques et financiers qui en résultent. En temps de crise, l'intérêt premier du politique est tourné de nouveau vers la croissance et l'emploi. Le PIB prend, fût-ce par défaut, une place 
centrale dans la détermination des budgets, dans l'élaboration des politiques économiques et fiscales et dans le calcul des déficits et des dettes publiques.

\section{Survie du mouvement}

Qu'ils perçoivent un «au-delà du PIB » comme un mouvement de mode ou comme porteur de conceptions radicalement nouvelles et potentiellement transformatrices, tous les acteurs rencontrés expriment des doutes ou des craintes quant à la longévité du mouvement. Outre les effets de la crise, l'importance d'un soutien politique et institutionnel est souvent mentionnée. À cet égard, certains acteurs suggèrent que, si les moyens alloués à l'élaboration de nouveaux indicateurs sont trop faibles, une certaine forme de pragmatisme pourrait influencer la production statistique, se fondant sur les indicateurs socioéconomiques existants.

\section{Crédibilité dominante des instituts de statistique}

Une plus grande confiance semble accordée aux indicateurs issus d'instituts officiels de statistiques qu'à ceux produits par les institutions associatives ou militantes (comme le World Wildlife Fund, par exemple). Ainsi, de nombreux acteurs interrogés font une distinction entre des indicateurs qu'ils considèrent comme neutres (issus des instituts de statistiques) et les indicateurs supposés servir un engagement.

\section{Absence de cadre réglementaire}

Pour de nombreux acteurs rencontrés, principalement parmi ceux qui sont impliqués, tant que la définition d'un « au-delà du PIB » ne sera pas encadrée par des règles et quel'usage de nouveaux indicateurs ne sera pas contraint par un mécanisme réglementaire, il sera difficile de faire d'un « au-delà du PIB » le concept fédérateur d'un quelconque changement. Il est suggéré de mettre en place une convention démocratiquement débattue et légalement contraignante en matière de bien-être (en la liant, par exemple, aux droits fondamentaux).

\section{Bien-être et soutenabilité : articulation complexe et non consensuelle}

L'absence d'un modèle macroéconomique alternatif fait écho à l'absence, plus profonde, de convergence d'intérêts et de valeurs sur l'articulation entre finalités humaines et environnement.

\section{Perspective cloisonnant bien-être et soutenabilité}

Tant au sein des ateliers que lors des entretiens, les représentations des enjeux économiques, sociaux, humains et écologiques sont cloisonnées. Il en résulte l'absence d'une perspective globale, qui semble prêter le flanc aux représentations existantes, où la dimension économique domine. Ainsi, les acteurs associant le bien-être à une notion psychologique et subjective voient dans la soutenabilité une affaire relevant exclusivement de l'environnement. Elle est un enjeu futur, souvent présentée en opposition avec le bien-être, considéré, lui, comme enjeu présent. Les acteurs en charge de domaines environnementaux sont nombreux à épouser cette perspective selon laquelle le maintien du bien-être dans le temps pourrait s'ancrer dans les modes de vie actuels, moyennant une évolution "favorable » du progrès technologique : c'est la croissance verte. Cette position est dominante parmi les acteurs institutionnels. Cette observation est corroborée par le fait que le bien-être vient peu à peu se substituer à la notion de soutenabilité dans les écrits officiels (Sébastien et Bauler, 2013).

\section{Perspective inclusive du bien-être et de la soutenabilité}

D'autres acteurs définissent le bien-être comme inextricablement lié à la nature. Dans cette perspective, la soutenabilité est définie comme le maintien, à travers les générations, des conditions qui assurent les libertés, au cœur desquelles se trouve un état naturel non détérioré. Les tenants de cette perspective de " soutenabilité forte " (Passet, 1979) sont plus favorables à des indicateurs de nature non monétaire et de type «empreinte». Cette position n'est pas dominante (nous l'avons rencontrée chez les quelques universitaires interrogés).

\section{Des pratiques peu participatives et recentrées sur l'économie}

Notre méthodologie ne nous a pas permis d'observer les pratiques effectives des acteurs. Nous abordons par conséquent celles-ci à travers leurs discours sur leurs pratiques effectives et potentielles. Les deux premiers types de pratiques identifiés font référence aux difficultés de donner à la participation un poids effectif dans l'élaboration des nouveaux indicateurs : ce sont l'échelle à laquelle l'indicateur est élaboré et les pratiques mêlant les approches bottom-up et top-down. Le troisième type de pratique traite des conditions d'adoption effective de nouveaux indicateurs. La quatrième pratique abordée est non effective et relate l'utilisation potentielle que certains acteurs entendent faire de nouveaux indicateurs.

\section{Échelle locale participative}

Si la participation effective des citoyens n'est pas aisée au niveau national, plusieurs réseaux participatifs ont fait preuve d'une importante proactivité au niveau territorial. 
Il apparaît de l'expérience de l'un de nos interviewés (universitaire) que l'échelle territoriale ou locale est un facteur essentiel à l'effectivité de la participation, tant parce que la proximité entraîne une plus grande implication citoyenne que pour des raisons purement organisationnelles. Au niveau institutionnel, il ressort que les appels à la participation citoyenne répétés aux échelles européennes et internationales ne semblent s'adresser qu'à un public d'experts déjà impliqués.

\section{Bottom-up vs top-down}

Concernant la participation à l'élaboration de nouveaux indicateurs, peu d'acteurs prônent l'unique participation des statisticiens ou la participation exclusive des citoyens. La majorité des interviewés favorisent la combinaison de processus bottom-up et top-down. Les processus bottom-up seraient orientés vers les citoyens pour recueillir leurs opinions et aspirations en matière de bienêtre et de soutenabilité, tandis que les démarches topdown seraient l'apanage des experts, qui quantifieraient les résultats de ces enquêtes sous forme d'indicateurs. Cette division du travail, en ne levant pas le voile sur les enjeux normatifs de tout processus de quantification (laissé ici aux mains des experts), soulève un enjeu démocratique que nous abordons dans la discussion.

\section{Conditions d'adoption effective de nouveaux indicateurs}

Il ressort de notre étude que les nouveaux indicateurs susceptibles de rencontrer un succès politique (en termes d'utilisation) devraient s'inscrire dans le sillage, ou du moins ne pas être en contradiction avec les méthodes de management en place et avec les objectifs de compétitivité, de rentabilité et d'innovation. Ces indicateurs doivent également être fondés sur une méthodologie liée aux conceptions et modes d'évaluation du modèle économique actuel (analyse coût-bénéfice, resource efficiency, etc.).

\section{Nouveaux indicateurs de bien-être et partenaires sociaux : entre profit et équité}

Les témoignages d'un petit nombre d'acteurs actifs dans le dialogue social ont mis en exergue une ambiguïté dans les effets potentiels de nouveaux indicateurs, s'ils devaient être utilisés dans la gouvernance des entreprises. Une certaine réticence semble s'observer au sein du patronat : certains craignent que de nouveaux indicateurs donnent une image négative de la situation des travailleurs, ce qui in fine conduirait à accroître la pression sur leur gestion de la firme. Parallèlement, une frange du patronat manifeste un intérêt pour ces nouvelles mesures : si le bien-être des travailleurs n'est pas exclusivement déterminé par le revenu, pourquoi ne pas agir sur les autres déterminants, si ceux-ci permettent de réduire le coût du travail, tout en maintenant, voire augmentant, le bien-être des travailleurs ? Dans ce cas, la mise en œuvre pratique de nouveaux indicateurs de bien-être aurait pour fonction de faire glisser l'attention des revenus vers d'autres variables. Les indicateurs deviendraient potentiellement le socle d'une nouvelle «paix sociale » qui irait de concert avec la logique de profitabilité.

\section{Analyse des discours : éléments de référentiel et lignes de conflits}

Quatre grands enjeux ressortent de manière «transversale » des savoirs, représentations et pratiques observés dans notre étude. Sur ces quatre enjeux se confrontent des visions dominantes et minoritaires. Afin d'évaluer si les débats sur ces enjeux révèlent un changement de référentiel, nous les passons au crible des quatre dimensions constitutives du référentiel, telles que définies par Muller (2010) : les "valeurs", qui sont les représentations les plus fondamentales sur ce qui est bien ou mal; les " normes », qui soulignent les écarts entre le réel perçu et le réel souhaité et définissent des principes d'action plus que des valeurs; les "algorithmes", qui désignent les relations causales exprimant une théorie de l'action («si..., alors... »), et les « images », vecteurs implicites de valeurs, de normes ou même d'algorithmes. Notons que le concept de référentiel n'est pas un simple ensemble d'idées, « dans la mesure où il exprime à la fois les intérêts et la vision du monde du groupe dominant au sein d'un système d'action publique » (Muller, 2010, p. 556). Le référentiel «ne constitue pas une vision parfaitement cohérente du monde, mais [...] balise le champ de perception de la réalité au sein duquel vont s'organiser les conflits » (Muller, 2010).

En amont des enjeux soulevés par les savoirs, représentations et pratiques observés, nous reviendrons en fin de section sur la distinction des communautés de pratiques que nous avons effectuée lors de notre récolte de données. Nous tenterons d'analyser a posteriori la pertinence de la distinction entre acteurs «impliqués » et «non impliqués » dans un « au-delà du PIB ».

\section{Cloisonnement des enjeux sociaux, humains, écologiques et économiques}

Les savoirs, représentations et pratiques observés dans notre étude révèlent que l'articulation des enjeux sociaux, économiques et écologiques reste partiellement impensée. Ainsi, des «valeurs » écologistes sont exprimées concomitamment à des «valeurs » matérialistes 
favorables à la croissance. La question des impacts de la croissance sur les déterminants du bien-être ou de l'environnement n'est abordée que par une minorité d'acteurs. Quand elle l'est, les acteurs y répondent par une importante confiance dans le progrès technologique. C'est le cas pour les tenants, majoritaires parmi les acteurs institutionnels, d'un principe «normatif» de «croissance verte », qui minimiserait les impacts de la production en termes de pollution et de consommation de ressources, sans remettre en cause le productivisme.

Cette focalisation sur le progrès technologique élude la possibilité de penser une révision structurelle des modes de production et de consommation et favorise des adaptations à la marge. Du cloisonnement des enjeux observé découle le maintien, chez la grande majorité des personnes rencontrées, d'un « algorithme » de type «si le PIB augmente, alors la situation est préférable, malgré ses imperfections ", notamment sur les plans de l'emploi et de la dette publique. Dans ce contexte, $l^{\prime}$ « image » rendue par le PIB reste pertinente pour la plupart des acteurs, puisqu'elle reflète l'évolution de ce qui reste considéré comme la source - certes incomplète - du bien-être en tant de crise : l'évolution de l'activité économique.

\section{Réformistes et révolutionnaires}

Les «valeurs » réformistes dominent nettement les discours. La grande majorité de ces « valeurs » visent une amélioration du modèle de croissance, par le biais, par exemple, d'améliorations technologiques, de la mise en œuvre de processus participatifs ou de l'utilisation $\mathrm{d}$ 'indicateurs de bien-être subjectif. Les acteurs justifient leurs positionnements par les urgences de la crise d'une part, et, par l'absence de modèle socioéconomique alternatif crédible d'autre part.

Plusieurs acteurs prônent, à cet égard, une meilleure utilisation d'indicateurs déjà existants. Un tel pragmatisme est susceptible de compromettre le potentiel d'innovation sociale d'un « au-delà du PIB ». Par ailleurs, l'absence de modèle alternatif crédible rend caduque, pour de nombreux acteurs, l'utilisation effective de nouveaux indicateurs. En termes de "normes", le réformisme qui domine les débats amène la majorité des acteurs à promouvoir l'accommodation du système existant. En temps de crise, cela signifie, pour beaucoup d'entre eux, renouer avec la croissance économique. L'« algorithme » qui prévaut alors repose sur la nécessité de la croissance : « en situation de crise, pas d'alternative possible». Un second « algorithme » concerne l'absence de cadre réglementaire : "tant que l'usage des indicateurs ne sera pas encadré réglementairement, ils ne seront pas effectifs ». En termes d' « images », la posture réformiste reste attachée au message que transmet le PIB sur l'état de la société, tout en étant consciente de ses limites en matière de bien-être.

\section{Temporalités conflictuelles}

Sur les questions de la définition du bien-être et de la soutenabilité, les "valeurs » écologistes et humanistes sont en friction. La majorité des acteurs considèrent en effet que le bien-être et la soutenabilité ont leurs temporalités propres et distinctes, le bien-être étant un enjeu présent, donc important et urgent, tandis que la soutenabilité est de l'ordre de la temporalité longue, donc moins urgent, bien qu'important. Les acteurs considérant, au contraire, qu'un mode de vie soutenable aujourd'hui est déjà source de bien-être présent, et que la soutenabilité est donc un enjeu urgent, sont très minoritaires parmi les acteurs rencontrés.

Corollairement à la prédominance des valeurs favorisant le bien-être à court terme, les « assertions normatives dominantes » donnent la priorité au bien-être, quitte à accepter dans certains cas que ses déterminants de court terme impactent le bien-être à long terme. La position normative selon laquelle il faut repenser nos modes de vie et les adapter aux contraintes écologiques est très peu présente dans le discours des acteurs institutionnels. Il apparaît, dans les discours dominants, que le moyen privilégié de concilier ces temporalités conflictuelles réside, encore une fois, dans le progrès technologique. L'un des « algorithmes » les plus courants est que « si l'on trouve la technologie appropriée, alors nos modes de vie actuels sont durables ».

En termes $\mathrm{d}^{\prime}$ « images », il ressort que le discours des acteurs institutionnels est dominé par une représentation de type "développement durable», dans laquelle les sphères économique, écologique et sociale sont trois cercles sécants, plutôt que par une représentation de type « soutenabilité forte », dans laquelle les sphères sont hiérarchisées - l'économie étant inscrite dans la sphère sociale, elle-même inscrite dans la sphère écologique. Nous avons observé, dans les critères de choix de nouveaux indicateurs, des tensions entre une perspective de long terme que requerrait une vision hiérarchisée d'une part, et les intérêts de court terme liés à l'agenda politique ou aux urgences économiques d'autre part.

\section{Entre démocratie et scientificité}

Un hiatus a été observé entre, d'une part, des « valeurs démocratiques » prônant la démocratisation des processus d'élaboration et de diffusion des indicateurs et, d'autre part, des "valeurs technocratiques » soutenant l'importance de confier l'élaboration des indicateurs aux experts, du fait de la grande technicité du sujet. De nombreux acteurs partagent ces deux familles de valeurs. En effet, la quasi-totalité des acteurs rencontrés prônent une élaboration des indicateurs mêlant grand public et experts, où le premier se prononcerait sur les dimensions à prendre en compte, tandis que les seconds les mettraient 
en chiffre. Si cette solution paraît consensuelle a priori, elle omet toutefois la question du contrôle démocratique de la quantification elle-même (Bruno et al., 2014 ; Cassiers et Thiry, 2011). Un seul des acteurs interrogés, un universitaire, a soulevé ce point crucial en matière de participation : une telle proposition normative, derrière la volonté d'intégrer le grand public aux débats, consisterait en réalité à donner aux experts un poids considérable dans la création de l'indicateur. Cette position est cohérente avec le grand crédit dont jouissent les instituts officiels de statistiques auprès des acteurs institutionnels rencontrés.

L'un des « algorithmes » les plus courants rencontrés sur la question du concepteur d'indicateur est que, « si un indicateur est produit par un institut officiel de statistique, alors il sera plus robuste qu'un indicateur produit par une ONG ». En termes d'« images », pour la majorité des acteurs, un indicateur issu d'un institut de statistique porte d'emblée une image de scientificité, qui laisse de côté la dimension normative de toute mesure.

\section{Quelle ligne de démarcation des communautés de pratique?}

La ligne de démarcation des communautés de pratique entre les acteurs « impliqués » et «non impliqués » transparaît-elle - au moins partiellement - à travers les savoirs et les représentations des acteurs rencontrés? De toute évidence, certains éléments de discours appartiennent explicitement à l'une ou l'autre communauté de pratique. On aurait tort toutefois d'en tirer la conclusion d'un lien explicatif entre participation ou non à un «au-delà du PIB » et positionnement axiologique ou normatif. Les nombreux éléments de discours communs aux deux communautés attestent de la porosité de cette frontière. Fautil alors chercher parmi d'autres facteurs les raisons de la faiblesse des savoirs et de la disparité des représentations ?

Concernant les savoirs, rappelons que les deux familles d'acteurs interrogées ont en commun d'agir dans des sphères institutionnelles où le PIB est un indicateur, pas forcément central, mais souvent interprété, utilisé ou mobilisé. S'il importe aux acteurs non impliqués dans un « au-delà du PIB » de défendre le maintien de l'utilisation de cet indicateur phare, on peut imaginer que ceux-ci aient développé un arsenal argumentatif à cet effet. De surcroît, ces acteurs, s'ils font régulièrement référence au PIB dans leurs pratiques, peuvent se trouver frontalement confrontés à ses limites. Il est dès lors étonnant que les critiques formulées à l'égard du PIB leur soient peu connues. La faiblesse de ces savoirs reflète-t-elle un manque de conscience professionnelle ou est-elle symptomatique d'une vision tronquée vis-à-vis d'un débat de société qui n'existe pas pour ceux vers qui il est principalement dirigé ? Quelle qu'en soit la raison, cette faiblesse des savoirs n'est pas l'apanage exclusif des acteurs non impliqués dans un « au-delà du PIB ». Nous avons vu que l'ensemble des acteurs interrogés montrait des savoirs superficiels - quand ils n'étaient pas nuls - à propos des alternatives au PIB.

Au vu de ce double phénomène (faiblesse des savoirs quant aux limites du PIB et à ses alternatives), il semble difficile d'associer la qualité des savoirs à un facteur motivationnel. Nous avançons l'hypothèse qu'une explication résiderait dans la façon dont les pratiques professionnelles, académiques et activistes se configurent : 1 'hyperspécialisation. Cette dernière rend de plus en plus ardue la construction de savoirs, que ce soit pour les acteurs impliqués ou non impliqués dans un « au-delà du PIB ».

L'hyperspécialisation pourrait également expliquer au moins partiellement - la disparité des représentations relevées au sein des deux communautés de pratique. Nous avons observé que les non initiés à un « au-delà du PIB » n'accordent que peu - voire pas - de crédibilité aux critiques du PIB. Pourtant, la plupart de ces limites sont actuellement formalisées au sein de la communauté d'économistes souvent reconnus par l'orthodoxie (voir Stiglitz et al., 2009). Que les partisans d'un « au-delà du PIB » soient perçus comme peu crédibles peut, là aussi, résulter de l'hyperspécialisation des instances institutionnelles chargées de la construction des indicateurs.

En somme, si les deux communautés de pratique que nous avions identifiées a priori pour collecter nos données ont effectivement des positionnements distincts en termes de valeurs, de normes, d'algorithmes ou d'images, ces positionnements peuvent toutefois être liés à des facteurs indépendants de leur adhésion ou non à l'objectif d'un « au-delà du PIB ». Les facteurs structurels, tels que l'hyperspécialisation, au sein des deux communautés de pratiques semblent aussi - sinon plus - importants à prendre en compte s'il s'agit de comprendre les raisons de l'adhésion à un « au-delà du PIB ».

\section{Conclusion}

Le concept de référentiel est souvent mobilisé pour analyser des politiques publiques précises: "l'émergence d'un nouveau cadre cognitif et normatif (constitué de nouvelles valeurs, de nouvelles injonctions normatives, de nouveaux algorithmes) constitue l'indice d'une transformation de la politique étudiée » (Muller 2005, p. 172). Nous transposons ici cette réflexion non pas à une politique précise, mais au cadre structurant qui sous-tend nombre d'entre elles : la croissance économique.

Nous constatons qu'au niveau des sphères institutionnelles, les débats sur un «au-delà du PIB », s'ils font entrer en ligne de compte de nouveaux enjeux, ne cristallisent pas l'émergence d'un cadre cognitif fondamentalement nouveau. Des valeurs cloisonnées, des normes dominées par l'impératif de croissance à court terme, des 
algorithmes inchangés et des images marquées par une vision qui ne questionne pas l'absence de hiérarchisation des sphères (environnement, société, économie) : ces différents éléments nous donnent à penser que les débats institutionnels sur un « au-delà du PIB », s'ils sont un lieu de construction de sens, ne contribuent pas à éroder l'invariant de la croissance économique. Il semble plutôt que l'intérêt des acteurs pour de nouveaux indicateurs relève plus d'une volonté ou d'une nécessité d'adapter les modalités de gestion publique ou les politiques publiques à de nouvelles contraintes, que d'une remise en question plus fondamentale du modèle productiviste sur lequel les économies sont bâties depuis plus de soixante ans.

Rien ne nous permet toutefois de conclure à un manque de volonté de changement ou, à l'inverse, à un volontarisme conservateur des acteurs en faveur du statu quo. Il apparaît que les nombreuses divergences et tensions qui traversent les débats nourrissent des ambiguïtés conceptuelles de fond. Ces dernières servent souvent une certaine forme de pragmatisme, qui s'inscrit dans les contraintes et les modes de fonctionnement de court terme du système. Tout comme pour le développement durable, les ambivalences liées à l'expression « au-delà du PIB » portent sur la signification de ce mouvement (si mouvement il y a), son origine historique (Conférence de l'UE «Beyond GDP » en 2007? Mouvement des indicateurs sociaux dès les années 1960 ? Les travaux des économistes Nordhaus et Tobin en 1972 ?), sa définition, son rapport à l'enjeu de soutenabilité environnementale, les objectifs sociaux et écologiques sous-jacents ainsi que son rapport à l'économie de marché capitaliste.

Évoquant le flottement du concept de «développement durable", Jacques Theys (2014) rappelle que « Matthias Maier (1999) avait avancé l'idée que cette ambiguiité conceptuelle pouvait avoir trois fonctions différentes : soit constituer [...] une couverture, permettant les manipulations tactiques [...] ; soit rendre possible la recombinaison des intérêts divergents, et ouvrir de nouvelles opportunités d'actions coopératives [...]; soit, enfin, préparer la transition vers un "nouveau" paradigme de développement ou de démocratie [...]». Concernant la première fonction, rien ne nous a permis d'identifier, dans les discours institutionnels, de formes de manipulation tactique. La fonction de « recombinaison d'intérêts divergents » en revanche nous semble se refléter dans les débats : les parties prenantes semblent en effet pouvoir s'accorder sur de nouveaux indicateurs ou sur la définition du bien-être ou de la soutenabilité, tout en s'opposant sur d'autres clivages, dont le clivage « gauche-droite ». Si cette observation peut interpeller, il serait toutefois hâtif de conclure à un basculement des clivages traditionnels vers de nouveaux axes d'opposition. À ce stade, nous pouvons uniquement pointer le risque d'une dépolitisation des débats (Dardot et Laval, 2010), précisément du fait de l'esquive du clivage « gauche- droite » au profit des aspects de bien-être et de soutenabilité. Ces derniers, moins « urgents » que les impératifs issus de la crise, peuvent constituer un moyen de détourner les discussions des enjeux de fond. Il semble en outre que les ambiguïtés conceptuelles qui traversent les débats sont susceptibles d'asseoir un rapport de force existant, tout en contribuant à nourrir l'illusion d'un débat démocratique sur des enjeux de société.

S'il s'agit de faire d'un « au-delà du PIB » le levier d'un changement de référentiel, plusieurs étapes cruciales semblent nécessaires. La première d'entre elles consisterait en une profonde clarification conceptuelle, qui mettrait systématiquement en exergue les fondements et impacts normatifs de chacun des positionnements observés dans les débats. Une telle clarification permettrait aux acteurs de cerner avec plus d'acuité leurs divergences et les potentiels intérêts conflictuels à l'aune desquels prendre position. Il importe par ailleurs de mieux rendre compte des urgences par rapport auxquelles les sociétés doivent trouver leur voie émancipatrice. Dans l'ensemble des débats, il s'agirait ensuite d'identifier les acteurs porteurs d'une vision cohérente des conflits et des problèmes à résoudre, ainsi que de leur articulation. De telles démarches clarificatrices des enjeux et des conflits, si elles ne peuvent prétendre résoudre ces derniers, contribueraient toutefois à l'élaboration d'un récit plus cohérent sur les finalités et moyens d'un « au-delà du PIB ». Elles permettraient également de mieux se positionner sur les indicateurs - nécessairement imparfaits - à privilégier pour baliser la transition vers une société post-croissance.

\section{Remerciements}

Cet article a grandement bénéficié de la lecture attentive et des conseils avisés de Philippe Roman. Merci également aux deux relecteurs pour leurs critiques et suggestions.

\section{Références}

Bruno I., Didier E., Prévieux J., 2014. Statactivisme : comment lutter avec des nombres, Paris, La Découverte.

Caminel T., Frémeaux P., Giraud G., Lalucq A., Roman P., 2014. Produire plus, polluer moins, l'impossible découplage? Paris, coédition Les Petits matins et Institut Veblen.

Cassiers I., Thiry G., 2011. Du PIB aux nouveaux indicateurs de prospérité. Les enjeux d'un tournant historique, in Cassiers, I. (Ed.), Redéfinir la prospérité. Jalons pour un débat public, Paris, Éditions de L'Aube.

Chancel L., Thiry G., Demailly D., 2014. Les nouveaux indicateurs de prospérité : pour quoi faire ? Study $\mathrm{n}^{\circ} 04$, Paris, Iddri.

Dardot P., Laval C., 2010. La nouvelle raison du monde. Essai sur la société néolibérale, Paris, La Découverte. 
Donnadieu G., Karsky M., 2002. La systémique, penser et agir dans la complexité, Rueil-Malmaison, Éditions Liaisons.

Gadrey J., Jany-Catrice F., 2012. Les nouveaux indicateurs de richesse, Paris, La Découverte.

Hák T., Janousková S., Abdallah S., Seaford C., Mahony S., 2012. Review report of beyond GDP indicators: categorisation, intensions and impacts. Final version of BRAINPOoL deliverable 1.1, a collaborative project funded by the European Commission under the FP7 programme (Contract no. 283024), Prague, CUEC.

Jackson T., 2010. Prospérité sans croissance, Bruxelles et Namur, De Boeck.

Maier M., 1999. The role of ideas in the politics of sustainable development, WGES Newsletter, 19, 7-11.

Meadows D.H., Meadows D.L., Randers J., Behrens III, W.W., 1972. The limits to growth: a report for the Club of Rome's project on the predicament of mankind, USA, Universe Book.

Méda D., 2000. Qu'est-ce que la richesse?, Paris, Flammarion.

Méda D., 2013. La mystique de la croissance : comment s'en libérer, Paris, Flammarion.

Muller P., 2005. Esquisse d'une théorie du changement dans l'action publique: structures, acteurs et cadres cognitifs, Revue française de science politique, 55, 1, 155-187.

Muller P., 1992. Entre le local et l'Europe. La crise du modèle français de politiques publiques, Revue française de science politique, 42, 2, 275-297.
Muller P., 2010. Référentiel, in Boussaguet L., Jacquot S., Ravinet P. (Eds.), Dictionnaire des politiques publiques, Paris, Presses de Sciences Po, 555-562.

Nordhaus W.,Tobin J., 1972. Is growth obsolete? in National Bureau of Economic Research, Economic research: Retrospect and prospect, New York, Columbia University Press, 5, 1-80.

Passet R., 1979. L'économique et le vivant, Paris, Payot.

Pestre D., 2011. Développement durable: anatomie d'une notion, Natures Sciences Sociétés, 19, 1, 31-39.

Sébastien L., Bauler T., 2013. Use and influence of composite indicators for sustainable development at the EU-level, Ecological Indicators, 35, 3-12.

Steffen W., Richardson K., Rockstrom J., Cornell S.E., Fetzer I., Bennett E.M., Biggs R., Carpenter S.R., de Vries W., de Wit C.A., Folke C., Gerten D., Heinke J., Mace G.M., Persson L.M., Ramanathan V., Reyers B., Sörlin S., 2015. Planetary boundaries: Guiding human development on a changing planet, Science, 347, 6223, DOI: 10.1126/science.1259855.

Stiglitz J., Sen A., Fitoussi J.-P., 2009. Rapport de la Commission sur la mesure des performances économiques et du progrès social, www.insee.fr/fr/publications-et-services/dossiers_web/ stiglitz/doc-commission/RAPPORT_francais.pdf.

Theys J., 2014. Le développement durable face à sa crise : un concept menacé, sous-exploité ou dépassé ?, Développement durable et territoires, 5, 1, http:// developpementdurable.revues.org/10196. 